\title{
EFEITO DO PH, ESPÉCIE E CONCENTRAÇÃO IÔNICA NA ABSORÇÃO DE ÁGUA DE HIDROGÉIS BIONANOCOMPÓSITOS CONSTITUÍDOS DE CMC/PAAM/LAPONITA RDS
}

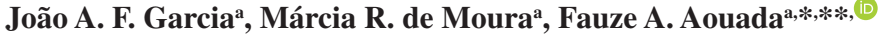 \\ aDepartamento de Física e Química, Faculdade de Engenharia de Ilha Solteira, Universidade Estadual Paulista "Júlio de Mesquita \\ Filho", 15385-000 Ilha Solteira - SP, Brasil
}

Recebido em 23/01/2019; aceito em 13/05/2019; publicado na web em 20/08/2019

\begin{abstract}
EFFECT OF PH, IONIC CONCENTRATION AND SPECIES ON THE ABSORPTION OF WATER BY HYDROGEL BIONANOCOMPOSITS CONSTITUTED FROM CMC/PAAm/ LAPONITE RDS. The objective of this paper was to study the effect of $\mathrm{pH}$, concentration and ionic species on the water absorption of the bionanocomposite hydrogels based on carboxymethylcellulose polysaccharide and laponite nanoclay. Bionanocomposite hydrogels were obtained via free radical polymerization. It was possible to observe that the swelling degree $(\mathrm{Q})$ and kinetic properties were strongly influenced, and controlled, by the presence and quantity of ionic species, decreasing their water uptake when compared to the same values obtained in distilled water. Furthermore, the addition of nanoclay decreased the water uptake sensibility of the hydrogel in ionic media. It was also observed that the presence of the nanoclay decreased the $\mathrm{Q}_{\mathrm{eq}}$ distilled water values from $32.5 \mathrm{~g} \mathrm{~g}^{-1}$ to $22.5 \mathrm{~g} \mathrm{~g}^{-1}$. The $\mathrm{pKa}$ variation of the bionanocomposites was a good indication of the possible interaction of the nanoclay-polymeric chains. The possibility of the control of water absorption (velocity and quantity) whereas these nanocomposites are $\mathrm{pH}$ - and salt-responsive may potentiate their application in agriculture. Agronomical studies are in progress, which can confirm its applicability in this area.
\end{abstract}

Keywords: nanoclay, swelling degree, kinetic properties, carboxymethylcellulose, pKa.

\section{INTRODUÇÃO}

Os hidrogéis são materiais relativamente novos, desenvolvidos inicialmente para aplicações médicas, entretanto, se tornaram foco de inúmeras pesquisas ao passar dos anos, expandindo suas aplicações em diferentes áreas como farmacêutica e agrícola. ${ }^{1}$ São materiais poliméricos que sofreram uma reação no qual suas cadeias adquiriram uma estrutura tridimensional, análoga a uma teia, chamado de reticulação. No caso de reticulações químicas, estas estão unidas por ligações covalentes (ligações primárias), formando assim um hidrogel do tipo químico ou permanente, não possibilitando que a reação de reticulação seja revertida. Há também a formação de hidrogéis do tipo físico ou temporário, gerados por pontos de interações físicas, ligações secundárias (como exemplo, forças de van der Waals e ligações de hidrogênio), formando uma rede tridimensional que pode ser dissolvida por estímulos externos, como variações de $\mathrm{pH}$, temperatura e soluções salinas., ${ }^{2,3}$

Os polímeros utilizados na formulação dos hidrogéis podem ser naturais, sintéticos ou semissintéticos. De maneira geral, os polímeros naturais apresentam biocompatibilidade, biodegradabilidade, e conferem aos hidrogéis, boas propriedades hidrofílicas, que é a capacidade de absorver água e/ou fluídos biológicos e liberá-los gradativamente, capacidade de sorção e dessorção de íons. ${ }^{4,5}$ Devido a estas propriedades, o polissacarídeo carboximetilcelulose (CMC) vem sendo utilizado na preparação de hidrogéis e seus nanocompósitos para diferentes aplicações tecnológicas. ${ }^{6-9}$ Por sua vez, o CMC é um polissacarídeo derivado da celulose, este considerado o polímero natural mais abundante encontrado na natureza. É utilizado em diversas atividades, como produção de detergentes, indústria têxtil e alimentícia devida sua propriedade de elevar a viscosidade. ${ }^{10}$

*e-mail: faouada@yahoo.com.br

** Grupo de Compósitos e Nanocompósitos Híbridos, Programa de PósGraduação em Ciência dos Materiais (PPGCEM), FEIS, Ilha Solteira - SP, Brasil
Com o objetivo de buscar uma sinergia dessas propriedades, muitas pesquisas embasam-se na formulação dos hidrogéis baseados em blendas ou compósitos poliméricos, além de argilas e nanoargilas, partículas magnéticas ou complexos iônicos. Dentre esses, apresentam-se hidrogéis constituídos de álcool poli(vinúlico)/carboximetilcelulose/óxido de grafeno e bentonita, ${ }^{11}$ alginato/poli(álcool vinílico)/laponita RDS, ${ }^{12}$ carboximetilcelulose/poliacrilamida/Cloisita $\mathrm{Na}^{+},{ }^{13}$ alginato/poli(ácido acrílico). ${ }^{14}$ Especificamente no caso de argilas, os cátions trocáveis presentes em suas galerias podem ser substituídos por cadeias poliméricas, ocorrendo a intercalação ou esfoliação. Além disto, podem ocorrer repulsões eletrostáticas entre as cargas negativas presentes na superfície das plaquetas da argila e os grupos aniônicos presentes nas cadeias poliméricas do hidrogel, o que pode provocar expansão de segmentos poliméricos, aumentado a capacidade de absorção de água dos nanocompósitos. De forma complementar, as argilas podem melhorar as propriedades térmicas, mecânicas e de sorção/dessorção de íons, e são relativamente de baixo custo, encontradas em abundância com um grande potencial para troca de íons, reduzindo o custo de produção de nanocompósitos. ${ }^{15}$

A laponita RDS é uma argila sintética da classe das laponitas, com fórmula química empírica $\mathrm{Na}_{0,7}\left[\left(\mathrm{Si}_{8} \mathrm{Mg}_{5,5} \mathrm{Li}_{0,3}\right) \mathrm{O}_{20}(\mathrm{OH})_{4}\right]_{0,7}$, possuindo estrutura similar a um disco cristalino com espessura na ordem de 1 $\mathrm{nm}$ e diâmetro de $30 \mathrm{~nm}$. Cada plaqueta de nanoargila, possui uma camada octaédrica com átomos de magnésio $(\mathrm{Mg})$ entre duas camadas tetraédricas de silício ( $\mathrm{Si}$ ). Pode ocorrer periodicamente a substituição dos átomos de magnésio por átomos de lítio (Li), causando uma deficiência de íons positivos que é contraposta pela presença de cátions de sódio $\left(\mathrm{Na}^{+}\right)$presentes nos espaços interplanares. ${ }^{16}$

Em recorrência das diferentes propriedades dos hidrogéis citadas anteriormente, os mesmos possuem diversas aplicações, como lentes de contato, ${ }^{17}$ tecidos artificiais, ${ }^{18}$ sensores e atuadores, ${ }^{19}$ tratamentos de câncer, ${ }^{20}$ substituição de cartilagens, tratamento de queimaduras, liberação controlada de fármacos, liberação controlada de pesticidas, entre outras. ${ }^{21,22}$ Em virtude da peculiar propriedade dos hidrogéis em absorver grandes quantidades de água em intervalos relativamente 
curtos e posteriormente liberar essa água de maneira mais lenta e controlada, agrega-se aos hidrogéis a possibilidade de carregar diversos componentes presentes em soluções aquosas. $\mathrm{O}$ que tem justificado a aplicabilidade de hidrogéis principalmente na área farmacêutica ${ }^{23-25}$ e na agricultura para liberação controlada de nutrientes e pesticidas. ${ }^{26-28}$ Especificamente na área agrícola, os hidrogéis vem sendo utilizados devido a sua biodegradabilidade, síntese de baixo custo e alta reprodutibilidade. O objetivo do trabalho foi compreender a influência de soluções salinas e pH na capacidade de absorção de água de bionanocompósitos constituídos de CMC/PAAm/nanoargila laponita RDS.

\section{PARTE EXPERIMENTAL}

\section{Síntese dos bionanocompósitos}

A síntese dos bionanocompósitos constituídos de carboximetilcelulose (CMC), poliacrilamida (PAAm) e nanoargila laponita RDS foi realizada por meio de polimerização via radical livre. ${ }^{1,13,22}$ As quantidades dos reagentes utilizados para reação foram obtidas em um estudo de otimização realizado anterioremente pelo nosso grupo de pesquisa GCNH. Desta forma, os diferentes hidrogéis foram obtidos utilizando $1 \% \mathrm{~m} / \mathrm{v}$ de CMC (Labsynth), $6 \% \mathrm{~m} / \mathrm{v}$ de acrilamida (AAm) (Vetec Química), 16,9 mmol L-1 de reticulador N'-N'-metilenobisacrilamida (MBAAm) (Vetec Química), 6,67 mmol L-1 de catalisador N-N-N'-N'tetrametiletilenodiamina (TEMED), 3,50 $\mathrm{mmol} \mathrm{L}^{-1}$ de iniciador persulfato de sódio $\left(\mathrm{Na}_{2} \mathrm{~S}_{2} \mathrm{O}_{8}\right)$ (Sigma). O componente variável da síntese foi a nanoargila, utilizadas nas proporções de 0 (referência), 1, 2, 4 e 5\% de laponita RDS em relação a soma das massas de CMC e AAm. A solubilização completa dos reagentes em água destilada foi realizada sob agitação magnética em temperatura ambiente. Antes da inserção do iniciador da polimerização, foi borbulhado gás nitrogênio $\left(\mathrm{N}_{2}\right)$ com o objetivo de retirar o gás oxigênio $\left(\mathrm{O}_{2}\right)$ dissolvido, minimizando a oxidação e maximizando a eficiência da polimerização. Em seguida, ainda sob agitação, o iniciador foi adicionado à solução, e esta foi rapidamente transferida para um molde de acrílico, permanecendo por 24 horas em temperatura ambiente.

Os hidrogéis, depois de polimerizados, passaram por uma etapa de purificação em água destilada por um período entre 7 e 10 dias, no qual a água foi substituída diariamente, com a finalidade de retirar os reagentes que não foram consumidos na reação. Uma ilustração do esquema da síntese dos bionanocompósitos é apresentada na Figura 1.

\section{Grau de intumescimento dos hidrogéis $(Q)$}

O grau de intumescimento (Q) é um parâmetro de extrema importância para avaliar e investigar as propriedades de absorção de água por materiais como os hidrogéis. A determinação dos valores de $\mathrm{Q}$ foi obtida pelo quociente da massa das amostras de bionanocompósitos intumescidas $\left(\mathrm{M}_{\mathrm{t}}\right)$ em um tempo t pela massa seca $\left(\mathrm{M}_{\mathrm{s}}\right)$, Equação $1{ }^{24}$

$$
Q=\frac{M_{t}}{M_{s}}
$$

A absorção dos bionanocompósitos foi avaliada em três diferentes meios de intumescimento, soluções salinas: cloreto de sódio $(\mathrm{NaCl})$, cloreto de cálcio $\left(\mathrm{CaCl}_{2}\right)$, e cloreto de alumínio $\left(\mathrm{AlCl}_{3}\right)$ em diferentes concentrações: 0,0125 a $0,20 \mathrm{~mol} \mathrm{~L}^{-1}$. Neste estudo foram utilizadas 2 composições de hidrogéis: amostras de hidrogel sem argila (referência) e $2 \%$ de nanoargila. O processo de absorção de água pelos bionanocompósitos foi também investigado em diferentes valores de $\mathrm{pH}$ (1 a 10), mantendo a força iônica constante $\left(\mathrm{I}=0,1 \mathrm{~mol} \mathrm{~L}^{-1}\right)$, utilizando as porcentagens de laponita RDS de 0 e $2 \%$.

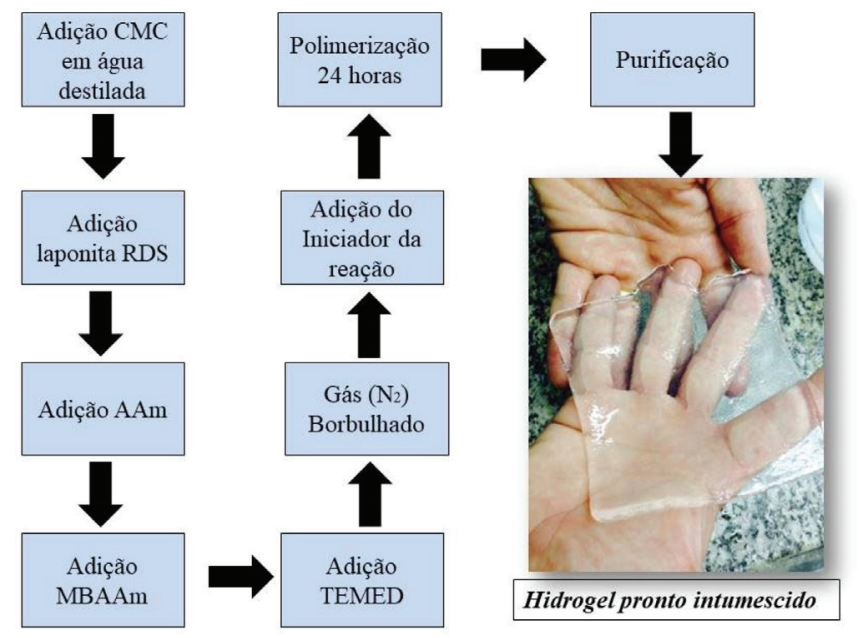

Figura 1. Esquema ilustrando o procedimento de síntese dos bionanocompósitos

Nesta metodologia, os hidrogéis purificados foram cortados em formado cilíndrico e secos em estufa com temperatura controlada de $40 \pm 1{ }^{\circ} \mathrm{C}$, até atingirem uma massa constante. Com o auxilio de uma balança analítica, foi determinada a massa seca $\left(\mathrm{M}_{\mathrm{s}}\right)$ das amostras. Em seguida, estas foram emergidas em frascos contendo $20 \mathrm{~mL}$ de solução. Em tempos pré estabelecidos (1, 2, 3, 4, 5, 6, 7, 8, 24, 30 e 48 horas), as amostras foram retiradas dos frascos removendo o excesso de água e determinadas as massas intumescidas $\left(\mathrm{M}_{\mathrm{t}}\right)$.

\section{Parâmetros cinéticos de absorção $n$ e $k$}

Os parâmetros cinéticos fornecem informações sobre a absorção de água pelos bionanocompósitos, sendo que o expoente difusional (n) descreve o tipo de mecanismo responsável pelo transporte de água para o interior do hidrogel. O coeficiente de difusão (k) está relacionado com a velocidade de absorção de água pelos hidrogéis em um determinado meio de intumescimento. Por meio da Equação 2, ${ }^{29}$ obteve-se os parâmetros cinéticos $\mathrm{n}$ e $\mathrm{k}$.

$$
\frac{M_{t}}{M_{e q}}=k \times t^{n}
$$

onde $\mathrm{M}_{\mathrm{eq}}$ é a massa do hidrogel intumescido no equilíbrio.

\section{RESULTADOS E DISCUSSÃO}

\section{Grau de intumescimento $(Q)$ em soluções salinas}

Inicialmente, utilizou-se solução de cloreto de sódio com concentrações entre 0,0125 e $0,20 \mathrm{~mol} \mathrm{~L}^{-1}$ e amostras de hidrogel com 0 e $2 \%$ de laponita RDS. Verificou-se a partir da Figs 2 a e 2 b que o meio de intumescimento influenciou a capacidade de absorção de água de ambos os hidrogéis, reduzindo consideralvelmente a quantidade de água absorvida, em comparação com valores de intumescimento em água. Os cátions de sódio $\left(\mathrm{Na}^{+}\right)$interagem com os grupos carboxílicos presentes nas moléculas de $\mathrm{CMC}$, formando complexos iônicos $\left(-\mathrm{COO}-\mathrm{Na}^{+}\right)$contribuindo para redução da repulsão eletrostática entre as cadeias poliméricas. Esta redução diminui o fenômeno de expansão das cadeias poliméricas formadoras dos hidrogéis e consequentemente a absorção de água. De acordo com Brito et al. $2013^{30}$ a redução da capacidade de intumescimento ocorre devido à capacidade de grupos carboxilato formar complexos inter e intramolecular com íons presentes na solução de intumescimento. 

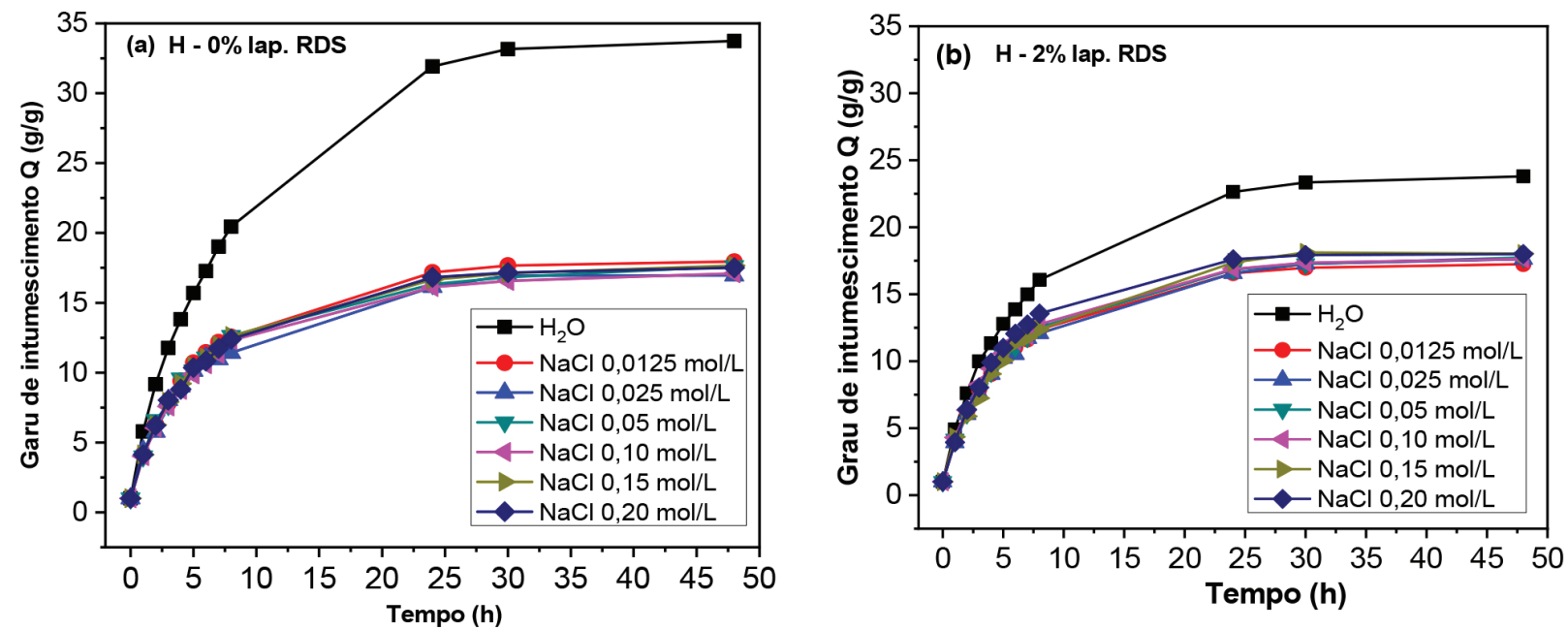

Figura 2. Grau de intumescimento em função do tempo para hidrogel sem nanoargila (a) e $2 \%$ de nanoargila (b) para água e diferentes concentrações de NaCl. pH da solução de intumescimento $\approx 5,5-6,0$
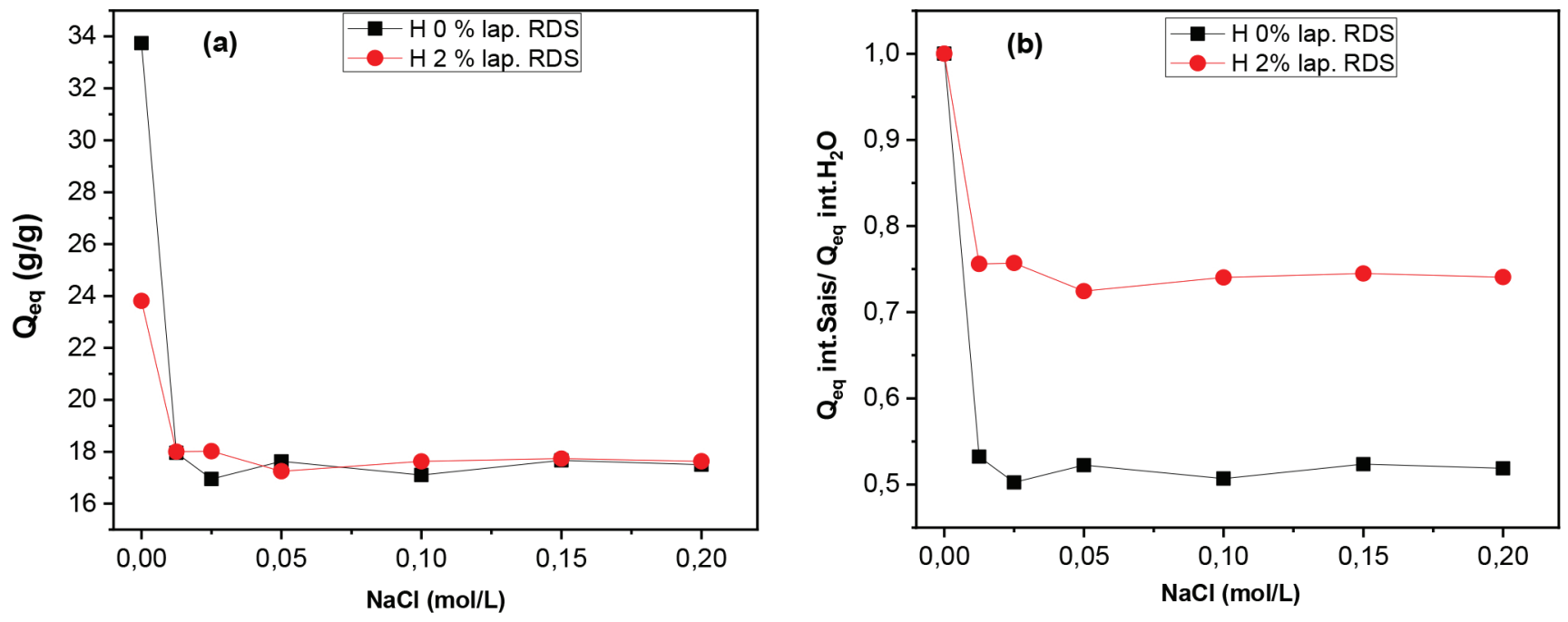

Figura 3. Grau de intumescimento no estado de equilíbrio $\left(Q_{e q}\right)(a)$ e razão do grau de intumescimento em sais $\left(Q_{\text {sais }}\right)$ pelo grau de intumescimento em água $\left(Q_{\text {ağua }}\right)(b)$ em função da concentração de $\mathrm{NaCl}$ para hidrogéis com 0 e $2 \%$ de nanoargila. $p H$ da solução de intumescimento $\approx 5,5-6,0$

Não foram observadas diferenças nos valores de $\mathrm{Q}$ ao variar as concentrações de $\mathrm{NaCl}$ (Figura 3a), ou seja, baixa concentração como $0,0125 \mathrm{~mol} \mathrm{~L}^{-1}$, é suficiente para ocorrer a saturação de possíveis pontos de interações dos grupos da CMC com $\mathrm{Na}^{+}$.

A Figura $3 b$ apresenta os resultados entre a razão do $Q_{\mathrm{eq}} \mathrm{em}$ meio salino por $\mathrm{Q}_{\mathrm{eq}}$ em água destilada em função da concentração de $\mathrm{NaCl}$. Verificou-se uma redução na absorção de água do hidrogel sem nanoargila em torno de duas vezes maior quando comparado ao hidrogel com $2 \%$ de laponita RDS. Isto indica que a adição da nanoargila reduziu a sensibilidade do hidrogel em responder ao estímulo externo, no caso, presença de sais. Pois a redução da absorção de água, quando imerso na solução de cloreto de sódio, foi menos acentuada. E que sse efeito pode estar relacionado à interação dos cátions de sódio com as plaquetas de nanoargila, formando complexos iônicos $\left(-\mathrm{O}^{-} \mathrm{Na}^{+}\right)$(Figura 4), diminuindo assim a interação com os grupos hidrofílicos da matriz, proporcionando uma maior mobilidade das cadeias poliméricas. ${ }^{15}$ Comparando os valores de Q em água, observa-se que a nanoargila reduziu a capacidade de absorção de água dos nanocompósitos. Isto ocorre devido a formação de pontos adicionais de reticulações entre as cadeias poliméricas. O que proporciona aumenta na densidade de reticulação, levando a diminuição da elasticidade do polímero. Comportamentos similares

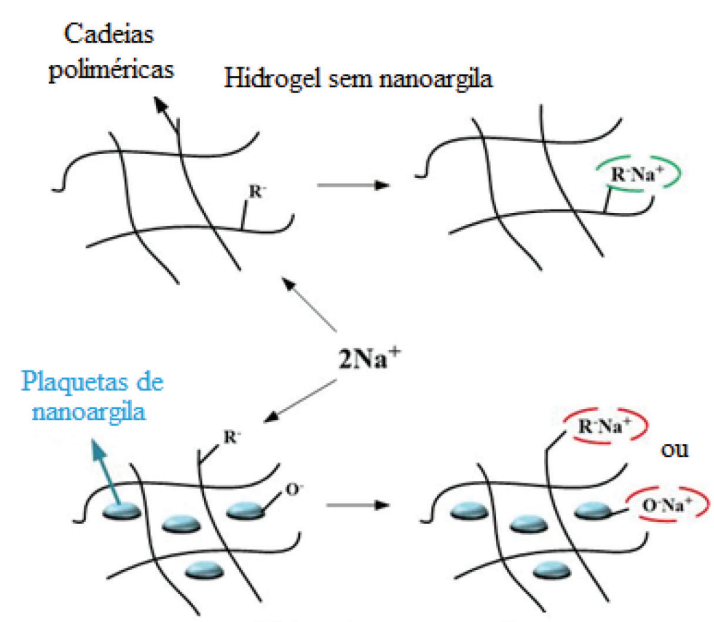

Hidrogel com nanoargila

Figura 4. Ilustração de uma possível interação entre os cátions de sódio $\left(\mathrm{Na}^{+}\right)$ da solução de intumescimento com grupos aniônicos da matriz polimérica e os grupos hidroxilas das plaquetas de nanoargila. $\mathrm{R}=\mathrm{OCH}_{2} \mathrm{COO}^{-}$. Ressaltase que por se tratar de interações coordenada-covalente, esta não ocorre somente em apenas 1 sítio como indicado na Figura 
foram observados em pesquisas anteriores desenvolvidas pelo nosso grupo de pesquisa em outros nanocompósitos. ${ }^{1,22}$

Paralelamente, realizou-se também o estudo de absorção de água dos mesmos bionanocompósitos em 2 dois outros meios salinos: cloreto de cálcio ou alumínio, ambos a $0,15 \mathrm{~mol} \mathrm{~L}^{-1}$. Observa-se a partir das Figuras 5a e 5b que a absorção de água decresceu nas soluções salinas comparado a quantidade absorvida em água destilada. Tal redução foi mais intensa conforme aumentou-se a valência dos cátions livres na solução, seguindo a ordem $\mathrm{Al}^{3+}>\mathrm{Ca}^{2+}>\mathrm{Na}^{+}$. Esse comportamento ocorre devido à grande quantidade de cargas positivas provenientes dos íons presentes na solução. ${ }^{31}$ Esses íons, na forma de cátions $\left(\mathrm{Al}^{3+}, \mathrm{Ca}^{2+} \mathrm{e} \mathrm{Na}{ }^{+}\right)$, interagem intra e/ou intermoleculares com os grupamentos $\mathrm{COO}^{-}$presentes na matriz do hidrogel, possibilitando a formação de pontos de interações físicas, reduzindo assim o fenômeno de expansão das cadeias poliméricas (Figura 6) e consequentemente a absorção de água. ${ }^{32}$ Como dito, esse fenômeno é mais influenciado quanto maior a valência do cátion presente na solução, o que explica a redução mais acentuada em cloreto de alumínio.

Outro fator que pode contribuir para a redução da absorção nas soluções salinas estudadas é a solubilidade dos sais. A $25^{\circ} \mathrm{C}$, os valores aproximados de solubilidade em água para as soluções de cloreto de alumínio, cloreto de cálcio e cloreto de sódio são 133 $\mathrm{g} / 100 \mathrm{~mL}, 74,5 \mathrm{~g} / 100 \mathrm{~mL}$ e $36 \mathrm{~g} / 100 \mathrm{~mL}$, respectivamente. ${ }^{33-35} \mathrm{O}$ fato da solubilidade do cloreto de alumínio ser maior está relacionado ao tamanho dos cátions quando estão dispersos na solução, sendo os valores de 1,02,1,00 e 0,53 ̊̊, para os cátions de sódio, cálcio e alumínio, respectivamente. ${ }^{36} \mathrm{O}$ tamanho dos cátions é importante, pois a probabilidade dos cátions $\mathrm{Al}^{3+}$ adentrar a matriz polimérica é maior, em virtude do seu tamanho e solubilidade, tendo, portanto, um efeito mais significativo, ${ }^{15}$ como demonstrado na Figura 5.

\section{Grau de intumescimento em soluções com diferentes valores de $\mathbf{p H}$}

Os estudos em soluções com diferentes valores de $\mathrm{pH}$ (1-10) demonstraram que ambas as amostras, com $2 \%$ argila e sem argila, tiveram comportamento semelhantes. Os bionanocompósitos se mostraram estáveis na faixa de $\mathrm{pH}$ (1-10), ou seja, não sofreram nenhum tipo de degradação visual ou estrutural durante o intervalo de 48 horas. Os valores de $\mathrm{Q}_{\mathrm{eq}}$ foram entre 17 e $18 \mathrm{~g} \mathrm{~g}^{-1}$ aproximadamente para os valores de $\mathrm{pH}$ entre 4-10. No entanto, as soluções com $\mathrm{pH}$ de 1 e 2 reduziram a capacidade de absorção de água de ambas as classes de hidrogéis para aproximadamente $14 \mathrm{~g} \mathrm{~g}^{-1}$ (Figuras 7a e 7b).

Com relação à Figura 8, observa-se o comportamento do grau de intumescimento no estado de equilíbrio $\mathrm{Q}_{\mathrm{eq}}\left(\mathrm{g} \mathrm{g}^{-1}\right)$ em função dos diferentes valores de $\mathrm{pH}$. O mesmo comportamento foi identificado para amostras com 0 e $2 \%$ de laponita RDS, onde o grau de intumescimento foi influenciado pelo $\mathrm{pH}$ da solução de intumescimento,
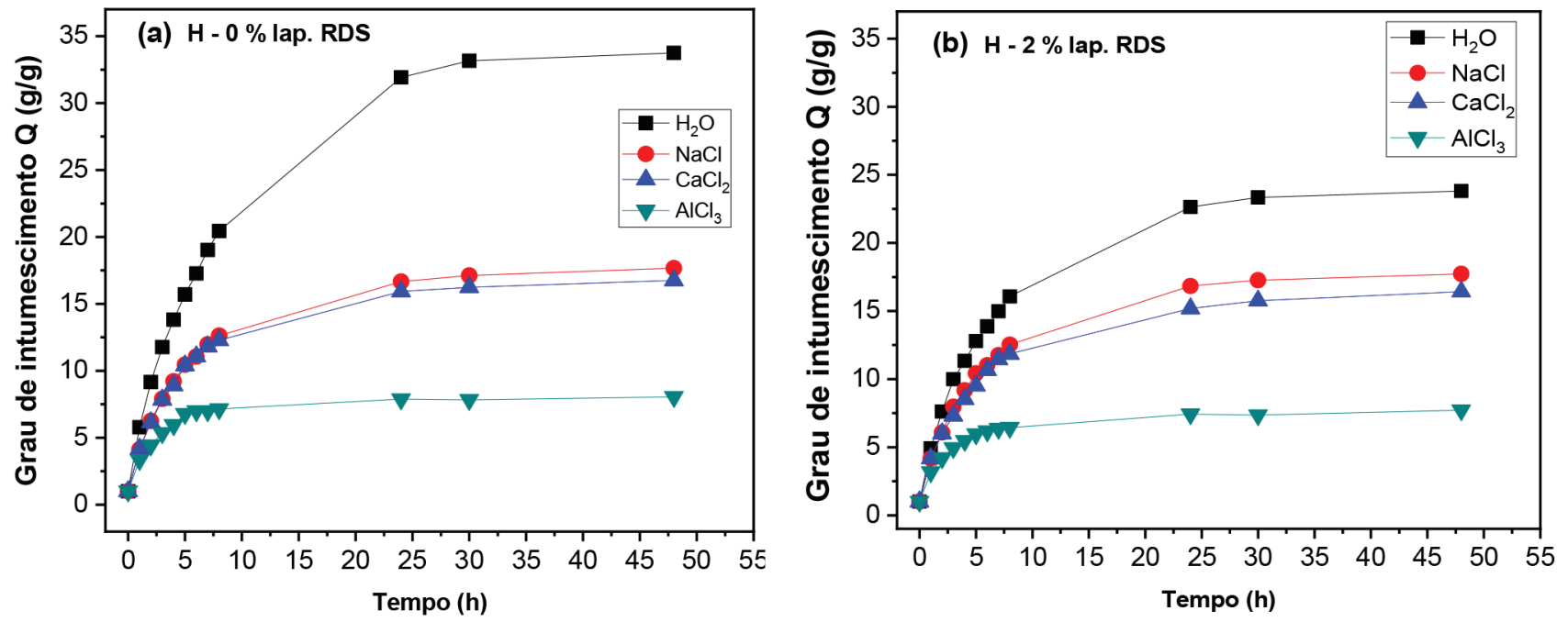

Figura 5. Grau de intumescimento em função do tempo para água e diferentes soluções salinas com hidrogel de 0\% de nanoargila (a), e hidrogel com $2 \%$ de nanoargila (b). [salina] $=0,15 \mathrm{~mol} \mathrm{~L}^{-1}$ e $\mathrm{pH}$ da solução de intumescimento $\approx 5,5-6,0$
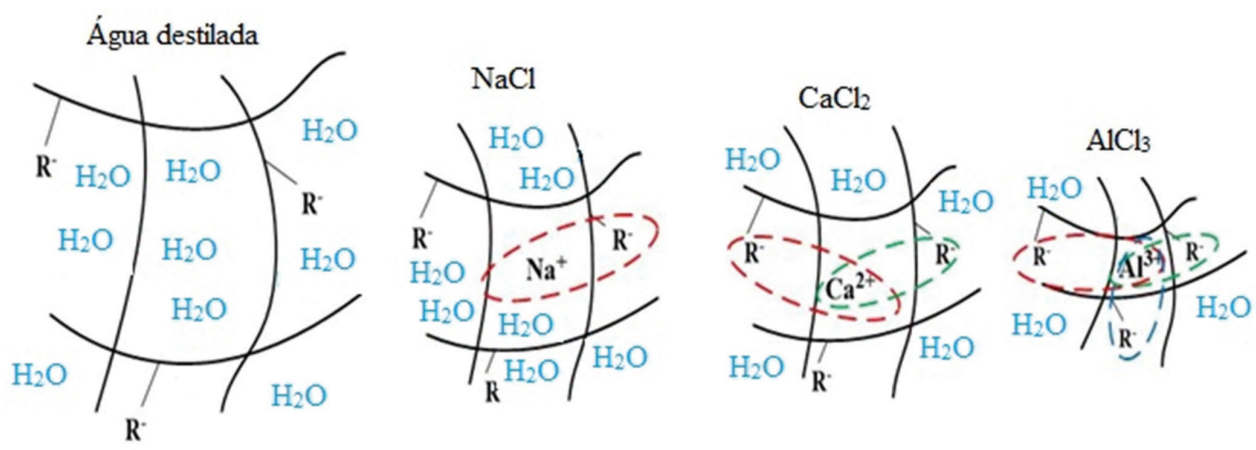

\section{Redução da absorção de água}

\section{Sendo $\mathrm{R}$ igual à $\mathrm{OH}$ ou $\mathrm{OCH}_{2} \mathrm{COO}^{-}$}

Figura 6. Representação da interação dos cátions de sódio, cálcio e alumínio com os radicais presentes na matriz dos hidrogéis constituídos de

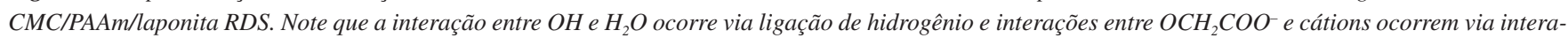
ções iônicas. Ressalta-se que por se tratar de interações coordenada-covalente, esta não ocorre somente em apenas 1, 2 ou 3 sítios como indicado na Figura 

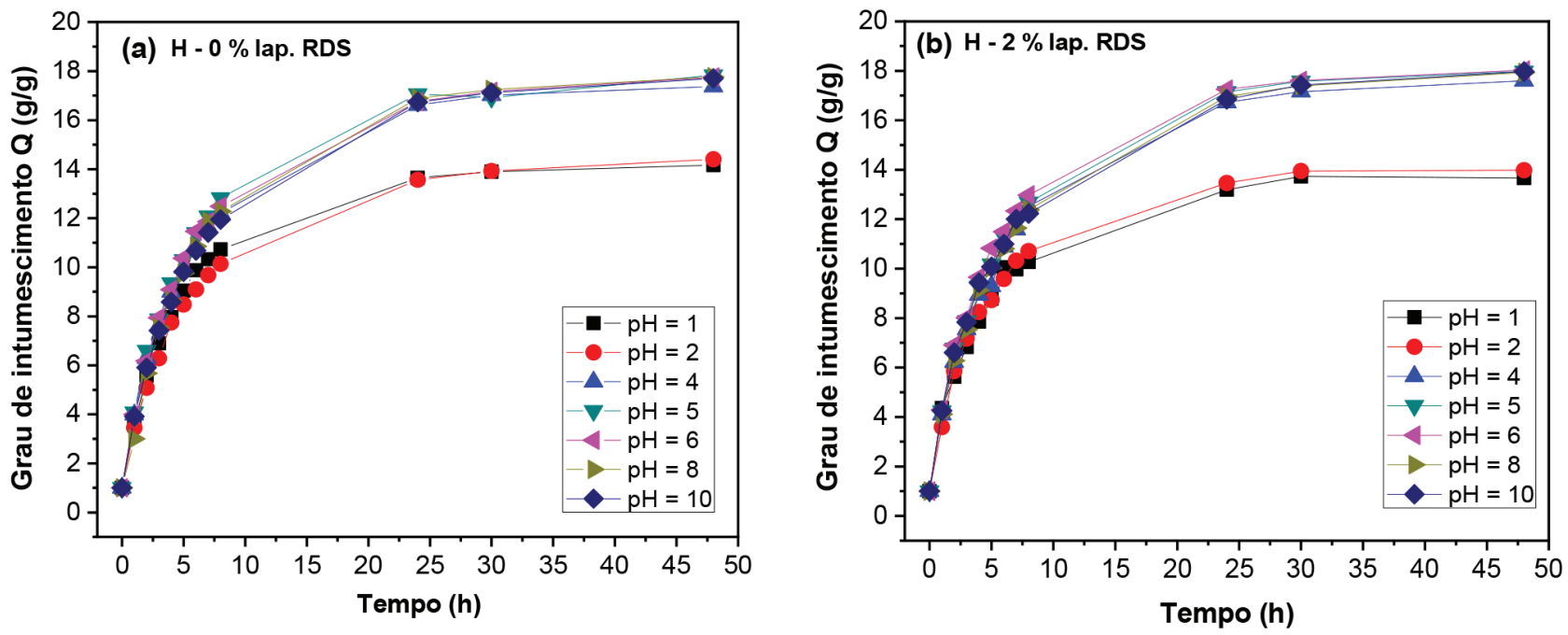

Figura 7. Grau de intumescimento $(Q)$ em função do tempo em soluções com diferentes valores de $p H$, para o hidrogel com $0 \%$ de laponita RDS (a) e hidrogel com $2 \%$ de laponita $R D S(b) . I=0,1 \mathrm{~mol} L^{-1}$

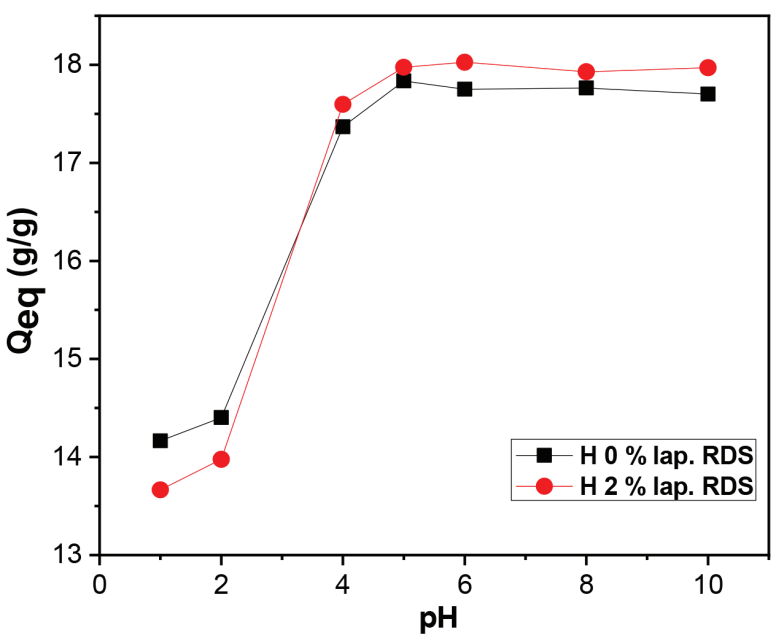

Figura 8. Grau de intumescimento no estado de equilíbrio $Q_{e q}\left(g^{-1}\right)$ para hidrogéis com 0 e $2 \%$ de nanoargila em função de soluções com diferentes valores de pH. $I=0,1 \mathrm{~mol} \mathrm{~L}^{-1}$

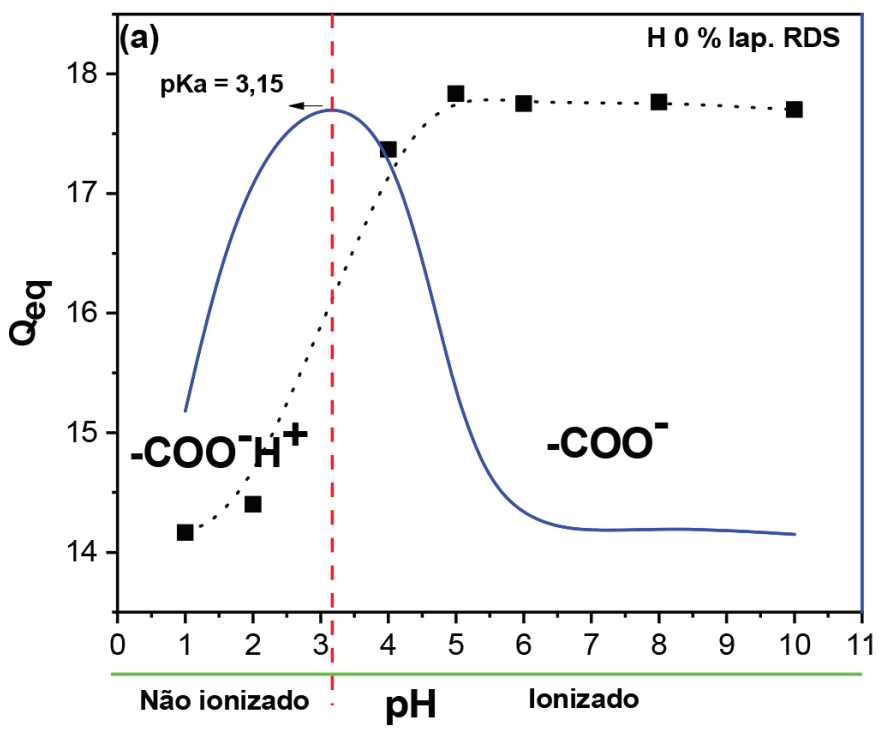

caracterizando-os como pH-responsivos. Ainda, a adição da nanoargila não influenciou o grau de intumescimento nas soluções de $\mathrm{pH}$ estudadas.

Ainda em relação à Figura 8, verifica-se um aumento brusco do grau de intumescimento ao variar o $\mathrm{pH}$ de 2 para 4, e mantendo-se praticamente constante em soluções com $\mathrm{pH}>5$. O menor grau de intumescimento para soluções em $\mathrm{pHs} 1$ e 2 pode estar relacionada com os valores de $\mathrm{pKa}=3,15$ (constante de acidez) dos grupos carboxílicos presentes na matriz de CMC. ${ }^{5}$ Nestas condições, o grau de intumescimento é menor devido a diminuição das forças repulsivas causadas pelos grupos carboxílicos quando estão ionizados, que ocorre em virtude da protonação dos mesmos $(\mathrm{COOH}),{ }^{2}$ resultante da maior concentração de prótons $\left(\mathrm{H}^{+}\right)$, conforme ilustrado na Figura 9. Já em $\mathrm{pH}>\mathrm{pKa}$, a presença dos grupos carboxílicos ionizados promove uma maior força de repulsão entre as cadeias poliméricas, aumentado a quantidade de espaços vazios e, consequentemente, aumentando a absorção de água. Além disso, a redução observada no valor do pKa do hidrogel com $2 \%$ de nanoargila, indica a interação da laponita RDS com os grupos carboxílicos presente na matriz, comportamento que corrobora com a redução da absorção em água destilada na concentração referida.

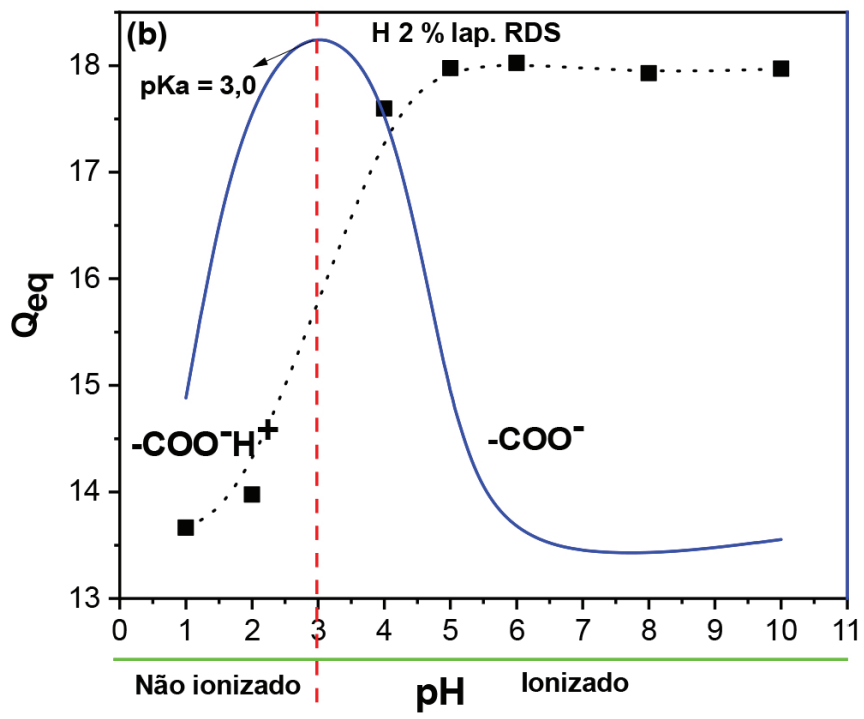

Figura 9. Derivada do grau de intumescimento no estado de equilíbrio $\left(Q_{e q}\right)$ para obtenção do valor de pka do hidrogel sem nanoargila (a) e hidrogel contendo $2 \%$ de laponita $R D S(b) . I=0,1 \mathrm{~mol} \mathrm{~L}^{-1}$ 


\section{Parâmetros cinéticos}

Os parâmetros cinéticos de intumescimento dos hidrogéis são extremamente importantes, pois facilita o entendimento do mecanismo de difusão de um dado solvente para o interior do hidrogel. Os valores de $n$ e $k$ dos hidrogéis em diferentes meios de intumescimento foram obtidos, por meio da Equação 2, utilizando os coeficientes angulares e lineares, respectivamente. Para valores de $n<0,45$, o mecanismo de difusão da água para o interior do hidrogel segue o mecanismo de difusão Fickiana. Quando $n$ é igual a 0,89 (transporte caso II), a difusão da água ocorre pelo mecanismo de relaxamento de cadeias, e quando $n>0,89$ o transporte denomina-se super caso II. Valores de $n$ variando entre $0,45 e 0,89$ indica a difusão por transporte anômalo, em que água difunde para o interior do hidrogel por relaxamento de cadeias e difusão. ${ }^{29,37}$

Pode-se observar a partir da Tabela 1 que os valores de $n$ em meio salino $\mathrm{NaCl}$ e $\mathrm{CaCl}_{2}$ para diferentes concentrações, tanto para as amostras sem nanoargila como para as amostras com $2 \%$ de laponita RDS, se aproximam de um comportamento anômalo. Uma redução nos valores de $n$ proporciona maior dificuldade na difusão de água para o interior do hidrogel. Este efeito que pode estar relacionado com a compactação das cadeias poliméricas, diminuindo sua expansão e consequentemente a absorção de água. Os menores valores de $n$ foram observados para o meio de intumescimento $\mathrm{AlCl}_{3}$. Indicando dificuldade de difusão das moléculas para o interior dos hidrogéis. Este fenômeno ocorre em virtude da alta concentração cátions trivalentes $\mathrm{Al}^{3+}$, podendo ser confirmado pelos valores de $\mathrm{Q}_{\mathrm{eq}}$ de 8,04 e 7,71 $\left(\mathrm{g} \mathrm{g}^{-1}\right)$ para hidrogéis com 0 e $2 \%$ de laponita RDS, respectivamente.

Da mesma forma, os valores de $k$ sofreram acréscimo na solução de intumescimento de $\mathrm{AlCl}_{3}$, ou seja, um aumento na velocidade de difusão. Verifica-se ainda um incremento da constante $k$ com o incremento com a concentração salina. Isto indica um aumento na velocidade de absorção de água para o interior dos bionanocompósitos. O que pode estar relacionado com a quantidade de cargas positivas dispersas na solução de intumescimento, proporcionando uma maior diferença de pressão osmótica entre a solução e a matriz polimérica. ${ }^{2,38}$ Tais resultados estão coerentes com os resultados de Q. O comportamento de $\mathrm{n}$ e $k$ em função das diferentes soluções salinas e água destilada é representado na Figura 10.

Por último, os valores de $n$ e $k$ obtidos em soluções com diferentes valores de $\mathrm{pH}$ apresentaram mecanismo de transporte anômalo no limite do transporte Fickiano. Não foi observado variação significativa dos valores de $n$ e $k$ para a faixa de $\mathrm{pH}$ estudada.

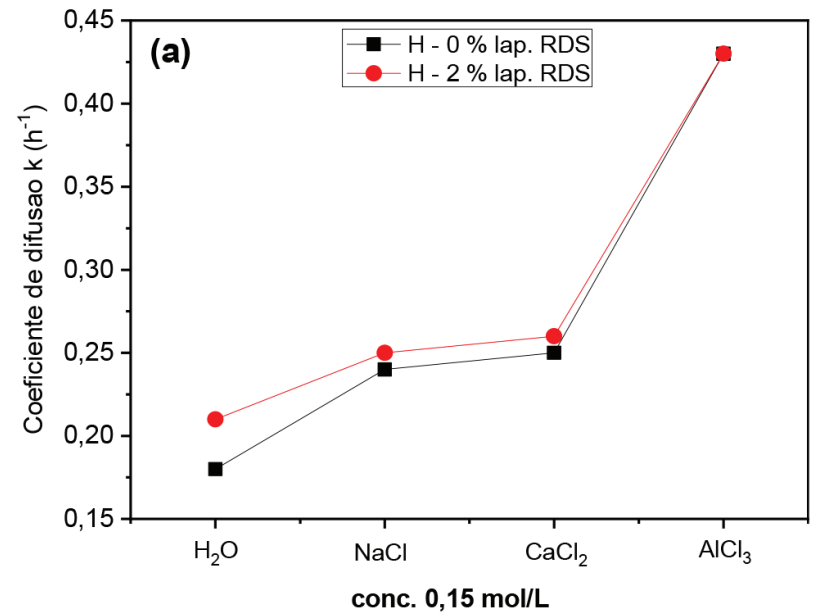

Tabela 1. Valores de $n, k, R^{2}$ e $Q_{e q}$ para hidrogéis com diferentes teores de laponita RDS e diferentes meios de intumescimento

\begin{tabular}{|c|c|c|c|c|}
\hline \multicolumn{5}{|c|}{ Meio salino } \\
\hline H 0\% lap. RDS & $\mathrm{n}$ & $\mathrm{k}\left(\mathrm{h}^{-1}\right)$ & $\mathrm{R}^{2}$ & $\mathrm{Q}_{\mathrm{eq}}\left(\mathrm{g} \mathrm{g}^{-1}\right)$ \\
\hline $0,0125 \mathrm{~mol} \mathrm{~L}^{-1} \mathrm{NaCl}$ & 0,54 & 0,24 & 0,992 & 17,96 \\
\hline $0,025 \mathrm{~mol} \mathrm{~L}^{-1} \mathrm{NaCl}$ & 0,51 & 0,26 & 0,971 & 16,95 \\
\hline $0,05 \mathrm{~mol} \mathrm{~L}^{-1} \mathrm{NaCl}$ & 0,53 & 0,24 & 0,981 & 17,63 \\
\hline $0,10 \mathrm{~mol} \mathrm{~L}^{-1} \mathrm{NaCl}$ & 0,53 & 0,24 & 0,997 & 17,47 \\
\hline $0,15 \mathrm{~mol} \mathrm{~L}^{-1} \mathrm{NaCl}$ & 0,54 & 0,24 & 0,994 & 17,66 \\
\hline $0,20 \mathrm{~mol} \mathrm{~L}^{-1} \mathrm{NaCl}$ & 0,53 & 0,24 & 0,992 & 17,50 \\
\hline $0,15 \mathrm{~mol} \mathrm{~L}^{-1} \mathrm{CaCl}_{2}$ & 0,53 & 0,25 & 0,994 & 16,75 \\
\hline$\underline{0,15 \mathrm{~mol} \mathrm{~L}^{-1} \mathrm{AlCl}_{3}}$ & 0,38 & 0,43 & 0,974 & 8,04 \\
\hline H $2 \%$ lap. RDS & $\mathrm{n}$ & $\mathrm{k}\left(\mathrm{h}^{-1}\right)$ & $\mathrm{R}^{2}$ & $\mathrm{Q}_{\mathrm{eq}}\left(\mathrm{g} \mathrm{g}^{-1}\right)$ \\
\hline $0,0125 \mathrm{~mol} \mathrm{~L}^{-1} \mathrm{NaCl}$ & 0,59 & 0,23 & 0,991 & 18,00 \\
\hline $0,025 \mathrm{~mol} \mathrm{~L}^{-1} \mathrm{NaCl}$ & 0,53 & 0,23 & 0,991 & 18,02 \\
\hline $0,05 \mathrm{~mol} \mathrm{~L}^{-1} \mathrm{NaCl}$ & 0,54 & 0,24 & 0,992 & 17,25 \\
\hline $0,10 \mathrm{~mol} \mathrm{~L}^{-1} \mathrm{NaCl}$ & 0,54 & 0,23 & 0,990 & 17,62 \\
\hline $0,15 \mathrm{~mol} \mathrm{~L}^{-1} \mathrm{NaCl}$ & 0,53 & 0,25 & 0,993 & 17,73 \\
\hline $0,20 \mathrm{~mol} \mathrm{~L}^{-1} \mathrm{NaCl}$ & 0,53 & 0,25 & 0,991 & 17,63 \\
\hline $0,15 \mathrm{~mol} \mathrm{~L}^{-1} \mathrm{CaCl}_{2}$ & 0,51 & 0,26 & 0,999 & 16,42 \\
\hline $0,15 \mathrm{~mol} \mathrm{~L}^{-1} \mathrm{AlCl}_{3}$ & 0,35 & 0,43 & 0,981 & 7,71 \\
\hline \multicolumn{5}{|c|}{ Soluções com diferentes valores de $\mathrm{pH}$} \\
\hline H 0\% lap. RDS & $\mathrm{n}$ & $\mathrm{k}\left(\mathrm{h}^{-1}\right)$ & $\mathrm{R}^{2}$ & $\mathrm{Q}_{\mathrm{eq}}\left(\mathrm{g} \mathrm{g}^{-1}\right)$ \\
\hline $\mathrm{pH}=1$ & 0,53 & 0,27 & 0,991 & 14,71 \\
\hline $\mathrm{pH}=2$ & 0,53 & 0,24 & 0,992 & 14,40 \\
\hline $\mathrm{pH}=4$ & 0,54 & 0,24 & 0,993 & 17,37 \\
\hline $\mathrm{pH}=5$ & 0,54 & 0,24 & 0,991 & 17,84 \\
\hline $\mathrm{pH}=6$ & 0,55 & 0,23 & 0,991 & 17,75 \\
\hline $\mathrm{pH}=8$ & 0,66 & 0,19 & 0,977 & 17,76 \\
\hline $\mathrm{pH}=10$ & 0,54 & 0,23 & 0,996 & 17,70 \\
\hline H 2\% lap. RDS & $\mathrm{n}$ & $\mathrm{k}\left(\mathrm{h}^{-1}\right)$ & $\mathrm{R}^{2}$ & $\mathrm{Q}_{\mathrm{eq}}\left(\mathrm{g} \mathrm{g}^{-1}\right)$ \\
\hline $\mathrm{pH}=1$ & 0,44 & 0,31 & 0,987 & 13,67 \\
\hline $\mathrm{pH}=2$ & 0,51 & 0,28 & 0,979 & 13,98 \\
\hline $\mathrm{pH}=4$ & 0,53 & 0,24 & 0,994 & 17,60 \\
\hline $\mathrm{pH}=5$ & 0,51 & 0,25 & 0,989 & 17,98 \\
\hline $\mathrm{pH}=6$ & 0,53 & 0,25 & 0,988 & 18,03 \\
\hline $\mathrm{pH}=8$ & 0,53 & 0,24 & 0,995 & 17,92 \\
\hline $\mathrm{pH}=10$ & 0,51 & 0,25 & 0,989 & 17,97 \\
\hline
\end{tabular}

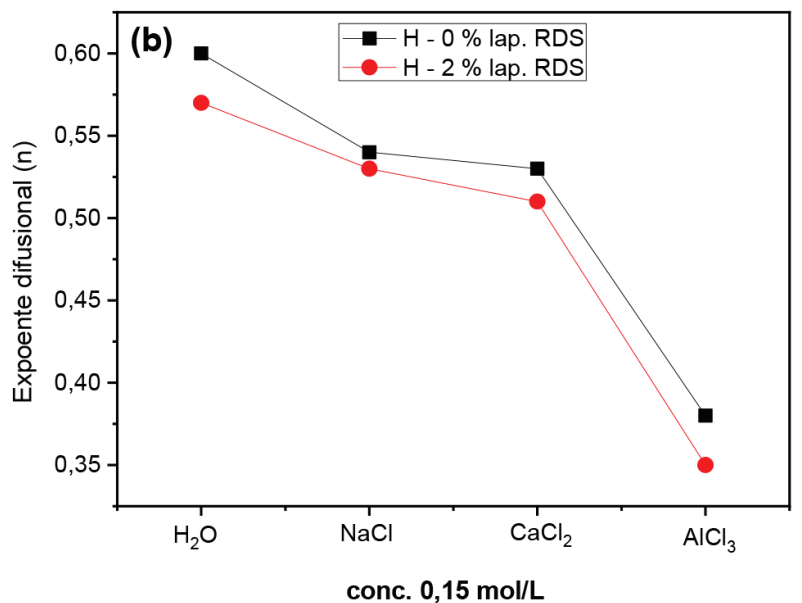

Figura 10. Coeficiente difusional $k(a)$, expoente difusional $n(b)$, em função de diferentes meios de intumescimento. pH da solução de intumescimento $\approx 5,5-6,0$ 


\section{CONCLUSÃO}

Foi possível realizar um estudo cinético de transporte de água de bionanocompósitos baseados em hidrogéis e nanoargila laponita RDS em diferentes soluções salinas e pHs. De maneira geral a presença de sais influenciou na absorção de água dos bionanocompósitos, diminuindo os valores do grau de intumescimento, comparado aos valores do estudo em água destilada. Isso ocorreu devido à presença dos cátions $\mathrm{Na}^{+}, \mathrm{Cl}^{2+}$ e $\mathrm{Al}^{3+}$, que interagem com os grupos aniônicos das cadeias poliméricas, diminuindo estatisticamente a probabilidade de que moléculas de água possam interagirem com tais grupos. Tais interações também reduzem o processo de expansão das cadeias poliméricas, reduzindo a absorção de água, causando redução nos valores de Q. Esse fenômeno é mais influenciado conforme se aumenta a valência dos cátions. Foi observado também que a nanoargila diminui os valores de Q em água, pois sua presença aumentou os pontos de reticulações entre as cadeias poliméricas.

Em relação aos estudos em soluções com diferentes valores de $\mathrm{pH}$, observou-se uma diminuição dos valores de $\mathrm{Q}$ em soluções com $\mathrm{pH}$ abaixo de $3(\mathrm{pH} \approx \mathrm{pKa})$, onde grande quantidade de prótons $\left(\mathrm{H}^{+}\right)$ interagem com os grupos aniônicos, impedindo parte da interação com as moléculas de água, além da expansão das cadeias poliméricas. Não foi observado nenhum tipo de degradação, visual ou estrutural, dos hidrogéis na faixa de $\mathrm{pH}$ estudada.

Em relação aos parâmetros cinéticos, foi observado que o meio de intumescimento influencia diretamente os valores de $n$ e $k$. Adicionalmente, em meio salino, a presença de cátions com maior valência ocasiona um aumento na velocidade de absorção; no entanto, promove uma redução nos valores de $n$, ou seja, maior dificuldade de absorção.

A possibilidade de controlar a velocidade e quantidade de água amplia a capacidade de aplicação dos bionanocompósitos desenvolvidos neste trabalho. Adicionalmente, estudos estão sendo realizados para testar sua aplicabilidade como materiais adsorventes de pesticidas assim como veículos carreadores de insumos agrícolas.

\section{AGRADECIMENTOS}

Os autores agradecem as agências de fomento Fapesp e CNPq pelo apoio financeiro. $\mathrm{O}$ presente trabalho foi realizado com apoio da Coordenação de Aperfeiçoamento de Pessoal de Nível Superior - Brasil (CAPES) - Código de Financiamento 001.

\section{REFERÊNCIAS}

1. Nascimento, D. W. S.; Moura, M. R.; Mattoso, L. H. C.; Aouada, F. A. J. Nanosc. Nanotechnol. 2016, 16, 821.

2. Aouada, F. A. Tese de Doutorado. Universidade Federal de São Carlos (UFSCar), São Carlos, 2009.

3. Bilici, C.; Karaarslan, D.; Ide, S.; Okay, O. Polymer, 2018, 151, 208.

4. Gyles, A. D.; Castro, D. L.; Silva, J. O. C.; Ribeiro-Costa, R. M. Europ. Polym. J. 2017, 88, 373.

5. Mahdavinia, G. R.; Mousanezhad, S.; Housseinzadeh, H.; Darvishi, F.; Sabzi, M. Carbohydr. Polym. 2016, 147, 379.

6. Singh, A.; Sarkar, DJ.; Mittal, S.; Dhaka, R.; Maiti, P.; Singh, A.; Raghav, T.; Solanki, D.; Ahmed, N.; Singh, SB. J. Appl. Polym. Sci. 2019, 136, 47332.

7. Chen, YS.; Long, YW.; Li, Q.; Chen, XH.; Xu, X. Int. J. Biol. Macromol. 2019, 126, 107.

8. Javanbakht, S.; Pooresmaeil, M.; Namazi, H. Carbohydr. Polym. 2019, 208, 294.

9. Godiya, CB.; Cheng, X.; Li, DW.; Chen, Z.; Lu, XL. J. Hazard. Mater. 2019, 364, 28.
10. Abd El-Mohdy, H. L. React. Funct. Polym. 2007, 67, 1094.

11. Dai, H.; Huang, Y.; Huang, H. Carbohydr. Polym. 2018, 185, 1.

12. Golafshan, N.; Rezahasani, R.; Esfahani, M. T.; Kharaziha, M.; Khorasani, S. N. Carbohydr. Polym. 2017, 176, 392.

13. Yonezawa, U. G. Dissertação de Mestrado. Universidade Estadual Paulista "Júlio de Mesquita Filho" (UNESP), Ilha Solteira, 2016.

14. Zheng, Y.; Huang, K.; You, X.; Huang, B.; Wu, J.; Gu, Z. Int. J. Biol. Macromol. 2017, 104, 1143.

15. Garcia, J. A. F. Dissertação de mestrado. Universidade Estadual Paulista "Júlio de Mesquita Filho" (UNESP), Ilha Solteira, 2018.

16. Lapasin, R.; Abrami, M.; Grassi, M.; Sebenik, U. Carbohydr. Polym. 2017, 168, 290.

17. Horne, R. R.; Judd, K. E.; Pitt, W. G. J. Drug Deliv. Sci. Technol. 2017, 41,410 .

18. Naahidi, S.; Jafari, M.; Logan, M.; Wang, Y.; Yuan, Y.; Bae, H.; Dixon, R.; Chen, P. Biotechnol. Adv. 2017, 35, 530.

19. Deng, K.; Bellmann, C.; Fu, Y.; Rohn, M.; Guenther, M.; Gerlach, G. Sens. Actuator B-Chem. 2018, 255, 3495.

20. Hu, C.; Liu, X.; Ran, W.; Meng, J.; Zhai, Y.; Zhang, P.; Yin, Q.; Yu, H.; Zhang, Z.; Li, Y. Biomaterials 2017, 144, 60.

21. Gyles, A. D.; Castro, D. L.; Silva, J. O. C.; Ribeiro-Costa, R. M. Eur. Polym. J. 2017, 88, 373.

22. Junior, C. R. F.; Moura, M. R.; Aouada, F. A. J. Nanosc. Nanotechnol. 2017, 17, 5878 .

23. Jesus, C. R. N.; Molina, E. F.; Pulcinelli, S. H.; Santilli, C. V. ACS Appl. Mater. Interfaces 2018, 10, 19059.

24. Ganguly, S.; Mondal, S.; Das, P.; Bhawal, P.; Maity, P. P.; Ghosh, S.; Dhara, S.; Das, N. C. Int. J. Biol. Macromol. 2018, 111, 983.

25. Fernandes, R. S.; de Moura, M. R.; Aouada, F. A. Quim. Nova 2018, 40, 60.

26. Nascimento, D. M.; Nunes, Y. L.; Figueiredo, M. C. B.; de Azeredo, H. M. C.; Aouada, F. A.; Feitosa, J. P. A.; Rosa, M. F.; Dufresne, A. Green Chem. 2018, 20, 2428.

27. Barbosa, D. H. O.; de Moura, M. R.; Aouada, F. A. Quim. Nova 2018, 41,380 .

28. Olad, A.; Zebhi, H.; Salari, D.; Mirmohseni, A.; Tabar, A. R. New J. Chem. 2018, 42, 2758.

29. Ritger, P. L.; Peppas, N. A. J. Control. Release 1987, 5, 37.

30. Tanaka, F. N.; Junior, C. R. F.; Moura, M. R. de; Aouada, F. A. J. Nanosci. Nanotechnol. 2018, 18, 7286.

31. Brito, C. W. de Q.; Rodrigues, F. H. A.; Fernandes, M. V. da S.; Silva, L. R. D. Da; Ricardo, N. M. P. S.; Feitosa, J. P. A.; Muniz, E. C. Quim. Nova 2013, 1, 40.

32. Li, X.; Li, Q.; Xu, X.; Su, Y.; Yue, Q.; Gao, B. J. Taiwan Inst. Chem. Eng. 2016, 60, 564.

33. NITROGENIUS, Produtos Químicos. Ficha de informação de segurança de produtos químicos. FISPQ. Cloreto de sódio, 2018. Disponível em: <http://www.hcrp.fmrp.usp.br/sitehc/fispq/Cloreto\%20de\%20S\%F3dio. pdf>. Acesso em: 11, fev. de 2018.

34. LABSYNTH. Ficha de informação e segurança de produtos químicos. FISPQ. 162. Cloreto de Alumínio. Última revisão 19/07/2010. Disponível em: <http://cloud.cnpgc.embrapa.br/wp-content/igu/fispq/laboratorios/ Cloreto\%20de\%20Aluminio.pdf>. Acesso em: 11, fev. de 2018.

35. TIOSERTEC. FISPQ n ${ }^{\circ}$ - 29, última revisão 15/04/2010. Cloreto de Cálcio. Filha de informação de produtos químicos. Disponível em: <http://www.hcrp.fmrp.usp.br/sitehc/fispq/Cloreto\%20de\%20Cálcio. pdf>. Acesso em: 11 fev. de 2018.

36. Callister Jr., W. D.; Rethwisch, D. G. Fundamentos da Ciência e Engenharia de Materiais, uma abordagem integrada. $4^{\mathrm{a}}$ edição. Editora LTC. 2014.

37. Ibrahim, S.; Nawwar, G. A. M.; Sultan, M. J. Environ. Chem. Eng. 2016, 4, 203.

38. Bortolin, A.; Aouada, F. A.; Longo, E.; Mattoso, L. H. C. Polímeros 2012, 22, 311 . 